\title{
LOS SABERES DE LA NEGACIÓN Y LAS PRÁCTICAS DE AFIRMACIÓN UNA VÍA PARA LA PEDAGOGIZACIÓN DESDE UNA PERSPECTIVA OTRA EN LA ESCUELA
}

\author{
KNOWLEDGE OF DENIAL AND REINFORCEMENT PRACTICES \\ AN OPPORTUNITY FOR A DIFFERENT PEDAGOGY AT SCHOOL
}

\author{
Ernell Villa Amaya* y Wilmer Villa**
}

\begin{abstract}
RESUMEN
Este texto es el resultado de un proceso de investigación que aborda los saberes de la negación, así como las prácticas de afirmación dentro de una relación escuela-comunidad, lo cual se hace a partir de la posibilidad que nos brinda la pedagogización, asumida como parte de una perspectiva otra. Se parte de la necesidad de generar una comprensión-acción que surge desde la identificación de problemas que afectan a la escuela y la comunidad. La investigación tiene un diseño flexible, en el que permanentemente se está volviendo sobre el proceso. La ruta metodológica se define a partir de la investigación cualitativa que busca interrogar las textualidades de los actores involucrados, en este caso la metodología en la indagación se desarrolla con base en lo etnográfico, lo narrativo y la investigación con-sentida que sitúa la acción en un relacionamiento horizontal.
\end{abstract}

Palabras clave: saberes de la negación, pedagogización, perspectiva otra, no-existencia, con-sentimiento.

\begin{abstract}
This text is the result of a research process that addresses the knowledge of denial and the reinforcement practices within a school-community relation, which is developed from the opportunity provided by pedagogy, assumed as part of a different perspective. It is based on the need to generate an understanding-action from identifying issues affecting the school and the community. The investigation emerges from a flexible design, where the process is permanently approached to. The methodological plan is defined from the qualitative research that seeks to question the culture of actors involved, as a semiotic text; in this case, the inquiry methodology is developed parting from the ethnographic and the agreed narrative research that places the action in a horizontal relation.
\end{abstract}

Keywords: Knowledge of denial, Pedagogy, a different perspective, Non-existence, Agreement.

Fecha de recepción: Febrero 20 de 2014 / Fecha de aceptación: Junio 20 de 2014

Tipología: Artículo de Investigación Científica y Tecnológica

Para citar este artículo: Villa, A, E., \& Villa, W. (2014). Los saberes de la negación y las prácticas de afirmación una vía para la pedagogización desde una perspectiva otra en la escuela. Praxis, Vol. 10, 21 - 36

*Doctor en Educación línea Estudios Interculturales Universidad de Antioquia, profesor Universidad de la Guajira Riohacha, Colombia. E-mail: evillaa@uniguajira.edu.co

${ }^{* *}$ Candidato a Doctor en Estudios Culturales Latinoamericanos. Profesor Proyecto Curricular Licenciatura en Humanidades y Lengua Castellana y Maestría en Investigación Social Universidad Distrital Francisco José de Caldas. Bogotá, Colombia. E-mail: villaw@hotmail.com 
"El saber cuenta cuando se encuentra lo que no se sabe" Ángel Bolívar Amaya

\section{INTRODUCCIÓN}

$\mathrm{E}$ ste texto es el resultado de una investigación que se viene realizando en la zona de Intracosta o Caribe Seco Colombiano, el cual se encuentra conformado por aquellos territorios que no son vecinos del mar pero que forman parte de la región que se nombra a partir de siete departamentos que están la norte del país. La pregunta de la cual parte este trabajo es la siguiente: ¿Qué papel desempeñan los saberes de la negación y las prácticas de afirmación como una vía para la pedagogización desde una perspectiva otra en la escuela, que contribuya a la construcción de la interculturalidad? Con base en esta formulación se buscó hacer una problematización de los referentes que configuran "lo dado por supuesto", es decir, el "ámbito del conocimiento seguro y no cuestionado” (Berger \& Luckmann, 1995, p. 87), el cual se puede asociar a las creencias que tienen las personas.

La postura que se adoptó para el desarrollo de esta investigación fue crítica, y se centró en la consideración del plano formal de la enunciación, o sea la parte discursiva, así como la práctica que anima a la acción entre las personas involucradas en el proceso. Desde ahí se trazó el propósito de indagar y actuar frente a los procesos que vinculan la escuela con la comunidad para propiciar nuevas emergencias que surgen de este relacionamiento, pero al momento de asumir esta posibilidad se tuvieron que afrontar los saberes de negación que operan en varias direcciones. Así mismo, se entró a significar las prácticas de afirmación cultural, las cuales pueden ser asumidas a partir de una pedagogización que se poya en una perspectiva otra en la escuela, sin dejar de lado los saberes y las iniciativas metodológicas propias. Esta investigación se ha venido desarrollando en los tres últimos años con pueblos étnicos del departamento del Cesar, donde se interroga no solo el espacio institucional de la escuela, sino los aspectos de la vida comunitaria que son pedagogizados en diferentes momentos y espacios.
La investigación partió de la generación de un espacio en común que aprovechó los encuentros para reflexionar y actuar en los aspectos que vinculan o desvinculan la escuela y la comunidad. En este sentido, se reflexionó sobre los modos de asumir la escuela y las formas de ser maestros y maestras; todo esto se encaminaría a la afectación de las prácticas en las escuelas, así como en la comunidad. Fue más el interés de generar un suelo epistémico y metodológico que conllevara la valoración de la experiencia desde los dos espacios que en algunos momentos se repelen y en otros se atrapan. A partir de esta búsqueda se establecieron las siguientes preguntas auxiliares:

- ¿Qué se entiende por saberes de la negación?

- ¿Desde qué referentes se pueden interpretar los saberes de la negación?

- ¿Cómo se expresan y se reproducen los saberes de la comunidad?

- ¿Desde qué referentes se pueden abordar las prácticas de afirmación en relación con el vínculo de la escuela y la comunidad?

- ¿Qué es la pedagogización?

- ¿Qué es una perspectiva otra?

- ¿Cuáles son los saberes que pueden ser pedagogizados en la escuela?

- ¿De qué se encarga la escuela y de qué se encarga la comunidad?

\section{Los saberes que cuentan desde la trayectoria de lo negado en educación}

Queremos empezar este apartado con la siguiente pregunta: ¿Qué es lo negado en educación a propósito de los referentes que actúan en la consideración de lo étnico y lo popular en Colombia? Se puede abordar esto a partir de los relatos de invención que se encuentran en los documentos históricos, los cuales han actuado en la configuración de un imaginario sobre los pueblos ancestrales. La configuración de un imaginario nacional se originó en la las apuestas de los criollos ilustrados, quienes desde un principio intentaron construir y sostener el proyecto de una nación monocultural, encarnada en lo andinocéntrico-criollo; a partir de esta referencia se buscaría alcanzar los rasgos civilizatorios que partían de la castellanización y la evangelización. 
Esto se vivió en toda América Latina, llegando en Colombia a adquirir unos matices singulares de producción y marginalización de franjas poblacionales. "En América Latina y el Caribe, la vehiculización de los relatos fundacionales ha sido posible a través de una intervención pedagógica que corresponden al interés de las élites nacionales de fijar los contenidos culturales que actuarían a favor de los procesos de sujeción de las personas a un marco de representación común” (Villa, 2013, p. 81).

El estudio de Sáenz, Saldarriaga y Ospina profundiza sobre los procesos de negación de lo étnico en el siglo XIX y principio del XX. Ellos analizan el proceso de modernización de la nación que sería un intento por higienizar y depurar las razas existentes para la época. Esto viene a constituir la materialización de un proyecto político, con el cual se buscaría superar el atraso, la ignorancia, la pobreza, la inmoralidad y la enfermedad de una "raza degenerada" que debía de ser — según las élites - intervenida. Con este fin se implementaría la eugenesia que partía de una explicación biológica de la raza, llegando a sostener el mejoramiento de la raza como una salida al problema. De ahí que los:

Pedagogos Reformistas como Miguel Jiménez López, Rafael Bernal Jiménez y Gabriel Anzola Gómez partían de una imagen pesimista del estado de la raza y de la infancia colombiana, pues las consideraban afectadas por profundas y gravísimas patologías físicas, mentales, morales y sociales, y por tanto privilegiaron opciones de reforma educativa de carácter remedial o "defensivo". Se conforma así un doble movimiento; por una parte, el salto a lo moderno, y por otra, detener el retroceso de la raza, su involución hacia un pasado primitivo y bárbaro. Inspirados en la teorías de la evolución de las especies, la preocupación central de estos intelectuales fue la biología de la raza. Según ellos, de su defectuosa conformación se derivaban los males intelectuales, morales y sociales del país (Sáenz, Saldarriaga \& Ospina, 1997, p. 11).
El contenido de este párrafo viene a reflejar el imaginario de la segunda mitad del siglo XIX, pero en nada difiere del pensamiento colonial que se apoyaba en la limpieza de sangre, que para Castro fue "el primer esquema de clasificación de la población mundial (...) el discurso de limpieza de sangre se tornó "mundial" gracias a la expansión comercial de España hacia el Atlántico y el comienzo de la colonización europea” (2010, p. 54). Todo se mantuvo igual y en nada cambió el tratamiento a los pueblos ancestrales por parte de los "criollos pioneros" (Anderson, 2007). A partir del estudio de estas continuidades se puede apreciar el "patrón de poder configurado” (Quijano, 2001, p. 120), manifestado en lo monocultural que contribuyó al establecimiento de lo nacional. Claramente esta operación forma parte de la colonialidad que, según Quijano, “es un patrón de poder configurado [...] que produciría unas tendencias de movimiento o desenvolvimiento histórico y de sus implicaciones en el largo plazo" (2001, p. 122).

La colonialidad que se presentó en la segunda mitad del siglo XIX y parte del XX se puede rastrear en el caso de la educación con los programas de “eugenización e higienización de la nación”, los cuales forman parte de la iniciativa de la clase política e intelectual por encontrar los caminos de la modernización. A lo largo de la historia colonial, posindependentista y moderna, se va a presentar este tipo de expresión política que incide en el tratamiento de los pueblos étnicos, así como las poblaciones populares. Todo esto viene a reflejar la "desconfianza en el pueblo" (Sáenz et al., 1997, p. 11) que las élites ejercieron desde el deseo de modernizar a la nación.

Para el caso del Caribe continental colombiano se formularon medidas "remediales" y "defensivas", con el firme propósito de atenuar el impacto del clima. A este respecto es importante recordar el papel que desempeñaría el determinismo climatológico, desde el cual se le atribuía al clima las causas de los males. Recordemos que "muy frio o muy caliente" igual a no humano, esto era lo que creían los griegos, "el hombre normal debía habitar el centro del mundo (Grecia, el Mediterráneo). A mayor alejamiento 
de esa región, la alteridad se hacía cada vez más manifiesta" (Boia, 1997, p. 40); esta creencia fue acogida por los intelectuales y los políticos andinocéntricos criollos. También se presentó el tropicalismo que generaría una calificación de las geografías del trópico, así como de las personas de esta parte del mundo.

El determinismo climatológico y el tropicalismo serían empleados como herramientas explicativas para justificar la exclusión de las dos costas colombianas. Desde ahí se representó a estas dos partes del país como el espacio geográfico donde vivían las personas atrasadas, incivilizadas e incultas. Supuestamente el causante de esto vendría a ser el clima malsano que existía en los litorales. Un dato que viene a reflejar esta consideración lo encontramos en un informe que reporta los índices de longevidad en la segunda mitad del siglo XIX, donde en el Estado Soberano del Magdalena la taza porcentual era muy baja respecto al resto de Estados; en este caso el número de habitantes que alcanzaban los 50 años eran muy pocos y todo se le atribuía al medio, en especial al clima.

En efecto, durante esa edad el porcentaje disminuyó, alcanzando tan solo el $6.4 \%$, lo cual demuestra que muy poco habitantes del Magdalena lograban superar medio siglo, seguramente por las condiciones de vida que predominaban en esas poblaciones y que de acuerdo con el geógrafo Vargas y Velazco eran "Un grupo de ranchos en torno de un pantano [...] lugares desapacibles, desiertos y enfermizos, donde nadie cumple allí 50 años" (Vergara \& Velazco citado por Alarcón, Calderón \& Santos, 2002, p. 27).

En el caso de la Costa Caribe Colombiana, esta también tendría un capítulo en lo que respecta a los programas de regeneración e higienización. Recordemos que unos de los creadores del programa político de la regeneración de la nación en el siglo XIX fue Rafael Núñez, un cartagenero que en varias veces fue presidente de la república. Ahora bien, frente al capítulo de la política de reducción de lo étnico-racial de la nación, queremos resaltar a Laureano Gómez, un político bogotano que en la primera mitad del siglo XX agitó el debate público sobre el rumbo de la nación. En su juventud, y siendo periodista, este personaje hizo un sobrevuelo sobre la geografía de la costa atlántica colombiana. Desde esta experiencia construyó una percepción deformadora de la región, la cual expresaría en una conferencia tiempo después. Esto pasó en la década del veinte del siglo pasado. Su conferencia se caracterizaría por ser descalificadora y con un tono racista.

La construcción de una percepción ligera sobre la gente de la costa atlántica calentaría más el agitado ambiente político, llevando a difamación y negación de las razas que habitaban esta parte del territorio nacional. Es de señalar que esta no era una postura individual, sino una postura de la dirigencia política e intelectual, que tenía una gran repercusión sobre el rumbo de la nación. Era una razón de Estado el asumir unas medidas de higienización y mejoramiento de la raza. Para esta época existían "los pesimistas" y "los melioristas” (McGraw, 2007). Los primeros guardaban una postura según la cual no había nada que hacer frente a las problemáticas que enfrentaba la nación, no había salida y el panorama era oscuro; en la segunda tendencia se presentaba un optimismo moderado, ellos decían que se podía hacer algo diferente, enfrentando la realidad del país. Es de señalar que tanto los unos como los otros utilizaban los mismos medios, es decir, el seguimiento a los planteamientos modernizantes que ayudaban hacer un diagnóstico de la problemática. "Tanto los pesimistas como los melioristas estaban de acuerdo en que el medio ambiente, el clima y la dieta tenía un papel significativo en el deterioro racial" (McGraw, 2007, p. 66).

Los contenidos que soportarían la razón de Estado se originaron en una élite política que impuso un lenguaje de negación y obliteración de la población étnica. La cuestión fue tan fuerte que una frase como "regeneración administrativa fundamental o catástrofe” de Rafael Núñez se convertiría en una razón de Estado, manifestada en una "política de gobierno" que llegó y se quedó durante mucho tiempo produciendo 
la vida, esto es "una política de control sobre la vida" (Castro, 2010). Estas disposiciones de la vida de los otros raciales con el paso del tiempo vendría a imponer la asimilación a "un patrón de poder configurado” y para esto se contaría con la educación.

Los agentes del estado y los reformistas locales se plantearon el reto de combatir la enfermedad y promover la salud moral y física. Al acoger una serie de prácticas basadas en un lenguaje moralista, que incluían la educación social, la purificación social y el control social, el movimiento a favor de la higiene convirtió la lucha contra la degeneración racial en un deber patriótico (McGraw, 2007, p. 66).

En las primeras décadas del siglo XX se impuso una actitud paternalista a través de la ayuda a las clases humildes, que se presentaban por parte de las élites como los causantes del atraso. Así que la clave fue adoptar medidas que a yudaran a remediar esos males. Fue así como se impuso un "lenguaje de los legisladores, la 'obra de civilización y de cultura, ya por lo que al decoro y la soberanía nacional se refiere' —esta frase forma parte de su defensa de una ley para limpiar Puerto Colombia- revela la convergencia del patriotismo hacia las clases trabajadoras de costeños con miras a mejorar la raza" (McGraw, 2007, p. 68). En esta dirección se identifican medidas como la mejora de la salud, así como los factores y situaciones que la producen; es el caso del consumo de bebidas fermentadas en el interior $y$, en la costa, los ambientes irregulares de los contextos urbanos. "Las élites de la Costa participaron con una actitud similar a la de los promotores de Santa Marta, quienes en 1926 definieron sus planes de traer el pavimento y el alcantarillado a la ciudad como una extensión de la ayuda de las "clases acomodadas" urbanas para levantar a las clases inferiores" (McGraw, 2007, p. 68).

Todas las manifestaciones se ven como parte de un plan de negación y producción de las desigualdades, mediante el cual se buscaba sacudir de la superficie cultural los elementos barbaros de una "etnia degenerada" o un "pueblo enfermo" que moralmente inviable. De ahí que la educación se convertía en una herramienta para enfrentar y deshacer todas esas manifestaciones negativas de la gente del trópico, gente estruendosa, ardiente y ociosa.

En la década de 1920, los congresistas que aprobaban la expansión de misiones de trabajo y educación invocaron doctrinas neo-lamarckianas al manifestar que en vista de su desastroso estado la región de la Costa no era apta para ser parte de la Nación, Estas tierras, decía el congresista Emilio Robledo, "se encuentran en la mayor ignorancia y es en ellas en donde es más frecuente la comisión de delitos y las violaciones del orden moral (McGraw, 2007, p. 69).

Los programas de higienización por medio de la depuración de la raza y la superación de la pobreza llevaron al establecimiento de mecanismos de control que fueron puestos en funcionamiento a partir de un ideal de superación de las deficiencias. Estos procesos de depuración cultural y social llegarían para quedarse en el pensamiento colectivo de la nación. Permanentemente se reactualizan y se producen discursos racistas, clasistas y regionalistas, que cobran diferentes matices, texturas, visualidades, sonoridades, en fin, ellos contribuyen a la invención de la vidas de los otros u otras, representados como cuerpos amenazantes, merecedores de todo control, tal como pasó en el caso de Cartagena, donde las medias extremas de higienización condujeron a la adopción de severos mecanismos, o con el "Departamento General de Saneamiento e Higiene” en 1912. “En Cartagena, hogares en barrios pobres se desinfectaron con el petróleo donado por la compañía de refinamiento de petróleo de Cartagena” (McGraw, 2007, p. 71). Los organismos que velaban por la higienización controlarían la "producción de la vida" en puertos, hospitales, mercados, pero sobre todo en escuelas, donde los niños y niñas eran obligados a adoptar un tipo de comportamiento que favorecía la adaptación al mercado. En cierta forma se emplearía la escuela como una instancia para disponer de la vida de las personas. De ahí 
que se necesitaría de un tipo de pedagogía que reforzara eso de producir la vida.

A continuación pasamos a abordar lo que sería una reacción a los saberes de la negación que se han expuesto en este apartado. Esta iniciativa se asume desde las prácticas de la afirmación del contexto y los actores, que en el contexto de la educación y la escuela se pueden abordar a partir de la pedagogización, la cual se convierte en una perspectiva otra, generadora de posibilidades abiertas que contribuyen a la conversación interepistémica. Esto se corresponde con la necesidad de descolonizar las prácticas y los discursos.

\section{La pedagogización una posibilidad de emergencia desde la "no-existencia"}

Para abordar la pedagogización iniciaremos con una diferenciación frente a lo que es la pedagogía, en la que encontramos que esta última se apoya en los desarrollos convencionales, los cuales han insistido en definiciones como “arte" (Eisner, 1995); la metodología que insiste en el "instruir la acción que constituye un sistema de educación” (Merani, 1985); “ciencia” (Herbart, 1935) y “disciplina en construcción” (Zuluaga, 1987). Estas consideraciones sobre lo pedagógico actúan en la visibilización del relacionamiento que existe entre los saberes y las prácticas, puestas en escena a partir de las mediaciones textuales que el profesor propone. Desde este referente vemos cómo la pedagogía ha estado asociada a la enseñanza y aprendizaje, donde estos referentes se pueden tomar como generalidades que inciden en la producción de lo pedagógico durante cierto tiempo. A este respecto, aquí no hacemos una definición porque esto implicaría hacer una diferenciación en las formas y los momentos como esta se asume.

Ahora bien, la pedagogización se puede asumir a partir de las experiencias localizadas, estos es, las múltiples voces, apuestas y disposiciones, que toman las posibilidades de "movilizar las acciones a través de procesos contextualizados, no se trata de una instancia funcional para la eficacia de la enseñanza aprendizaje [...] se trata de ver la pedagogía-accionada y accionadora de procesos políticos, culturales y situacionales, llegando a superar las concepciones de la educación y la escuela como un microespacio o microcosmo asilado de la realidad contextual" (Villa \& Villa, 2010, p. 79).

El hecho de asumir la pedagogización nos lleva a valorar las experiencias en los contextos, así como los actores; se trata de tomar las trayectorias y las configuraciones que inciden en la orientación y articulación de las propuestas, los contenidos y los escenarios, donde las personas se relacionan desde una afectación mutua. Frente al ejercicio de nombrar lo que consideramos como pedagogización, aclaramos que no se trata de hacer un acto donde se pasa a otra denominación, exaltando lo novedoso. Por supuesto, no se trata de hacer un tipo de esnobismo categorial, establecer una analítica anfibia y camaleónica, conducente a lo que en otras ocasiones hemos llamado más de lo mismo a través de la exaltación de nuevas palabras.

La pedagogización forma parte de una apuesta política que empieza con la práctica del nombrar desde, con, entre y para los contextos y las personas. A partir de ahí se piensan, se buscan, se justifican, se construyen y se proyectan los fines o propósitos de la educación, así como los ambientes donde se escenifican-negocian y problematizan los contenidos, las metodologías, las estrategias, los dispositivos y los resultados. Unas de las intenciones que mueve la pedagogización en tanto proceso político es conseguir la soberanía intelectual del pueblo, comunidad, grupo y nación; esto a partir de la valoración $y$ en reconocimiento de una co-existencia de diferentes instancias de producción. Consiste en asumir un relacionamiento más horizontal, donde el ver, el nombrar y el actuar se hace y se deshace de acuerdo con las posibilidades de co-creación o coparticipación direccionadora de la experiencia.

La pedagogización actúa desde una localización precisa, es decir, desde adentro y desde abajo. En este sentido, la pedagogización se afirma en la necesidad de interrogar los lugares a partir de los cuales se producen las representaciones y las prácticas. Es más la necesidad de preguntarse: 
¿quién representa a quién?, ¿desde dónde lo hace? y ¿bajo qué situaciones se produce la representación?

La pedagogización se vive como un proceso que se construye y no como lo que está dado, así como lo que se impone. De ahí que la pedagogización parte de la motivación que lleva a la conversación y el relacionamiento horizontal. Esto se puede ver por ejemplo en una dinámica de diálogo intergeneracional, donde los mayores orientan la conversación sin generar desconocimiento de las y los jóvenes; como se dijo, es un proceso de mutua afectación. Por esto, se parte de tomar en serio aquellas experiencias y actores que han sido invisibilizadas a lo largo de la historia. La pedagogización reacciona contra la producción del "pensamiento abismal moderno" que establece una localización que deja a unos por dentro y a otros por fuera. Santos (2009) a este respecto sostiene:

El pensamiento occidental moderno es un pensamiento abismal. Éste consiste en un sistema de distinciones visibles e invisibles, las invisibles constituye el fundamento de las visibles. Las distinciones invisibles son establecidas a través de líneas radicales que dividen la realidad social en dos universos, el universo de "este lado de la línea" y el universo del "otro lado de la línea". La división es tal que "el otro lado de la línea” desaparece como realidad, se convierte en no existente, y de hecho es producido como no-existente. Noexistente significa no existir en ninguna forma relevante o comprensible de ser. Lo que es producido como no-existente es radicalmente excluido porque se encuentra más allá del universo de lo que la concepción aceptada de inclusión considera es su otro (p. 160).

Como resultado del "pensamiento abismal moderno" se produce una demarcación fija, la cual se manifiesta en el dominio del pensamiento, pero se manifiesta en la organización del mundo social. En el caso de la escuela se producen ciertas localizaciones de cuerpos que son dejados de lado, por ejemplo las niñas y niños ciegos, los sordos, las mujeres, los afrocolombianos, los indígenas, los marginales urbanos que provienen de otras regiones del país o los desplazados por el conflicto armado. Sobre ellas y ellos recae una demarcación correspondiente al "pensamiento abismal moderno". Esta se hace presente en las formas de clasificar, controlar, disponer del espacio, los cuerpos y las prácticas de las personas. Con base en la pedagogización se desarrolla una repuesta a la marginalización de las experiencias que no se encuentran del lado de lo visible que gobierna el "pensamiento abismal moderno". La posibilidad de actuar en escenarios concretos de acuerdo con la pedagogización se ve en la necesidad que las personas se conviertan en actores de sus propias iniciativas, bajo la autonomía o también bajo la "co-alianza”. Desde ahí podemos decir: se trata de una conexión con lo desvalorizado, lo negado, lo silenciado y dejado por fuera de cualquier posibilidad de ser representación. En este caso, la pedagogización dispone, insiste y se interesa por aquellas instancias de producción "deformadas, desacreditada y desalojadas" (Villa, 2014a), esto es, habitar o ser parte de "lo que ya no tiene caso insistir" (Villa, 2014b). La pedagogización en cierto modo se encarga de la "no-existencia", "la no-localización", "la no-valoración”, para, desde ahí, asumir nuevas posibilidades que parte de los encuentros y re-encuentros; tal como pasaría con la desconsideración de la oralidad en la escuela, que vendría a encarnar lo "no-existente" porque en la escuela lo central son las lecturas y las escrituras; la oralidad, en este caso, la convierten en la otra, la extraña, la intrusa, la no invitada al aula, esa que lleva a los niños a salirse de su propio autocontrol en el uso de la palabra.

\section{El desafío de asumir una perspectiva otra en la escuela}

Hablar de otra perspectiva no es lo mismo que hablar de una perspectiva otra; la diferencia entre la primera y la segunda se establece a partir de la localización de una experiencia que sigue la trayectoria establecida. Esto es una derivación que se manifiesta en la coexistencia de tendencias, perspectivas, enfoques, corrientes, escuelas y autores, que continúan o manifiestan ciertas incompatibilidades, pero dentro de un mismo 
tronco epistemológico, el cual resulta de una geopolítica del conocimiento que es operado a partir de unos centros hegemónicos que intervienen en la producción, la formalización, la legitimación, la circulación y la distribución del conocimiento en el ámbito mundial. En este caso, los autores que forman parte de otra perspectiva son los que gozan de la promoción y el respaldo del "imperialismo impreso", en los ámbitos internacional, regional, nacional y local; para estos autores no existen las fronteras o los limites porque sus elaboraciones son celebradas como si fueran válidas para todo tiempo y contexto.

Al momento de establecer unos referentes de lo que es otra perspectiva en el plano de lo epistemológico, tenemos autores como Karl Marx, Friedrich Nietzsche, Edmund Husserl, Theodor Adorno, Michel Foucault y Jean-François Lyotard, entre otros que forman parte del grupo de autores que el profesor Grosfoguel (2006) denomina como pensamiento "blanco-disidente", pero que su "corpo-política” procede de una demarcación geográfica controlada desde los centros hegemónicos de poder imperial. Asumimos la “corpo-política” como una configuración de una corporalidad que construye sus elaboraciones de un referente político que se vuelve sostén del lugar de enunciación o el lugar desde el cual sustenta su discurso; este incide, pero desde un lógica de producción eurocéntrica se niega o se esconde. A este respeto, Grosfoguel (2006) sostiene:

El punto central de las perspectivas epistémicas otras es la relación estrecha entre el lugar de enunciación, esto es, la localización geopolítica y corpopolítica del sujeto del sujeto que habla en las coordenadas del poder global y sus estrecha relación con la producción de conocimiento. Las perspectivas otras nos ofrecen la posibilidad de «corregir» y «redefinir» los límites de la producción de conocimiento que procede de la mirada eurocentrada (p. 151).

Ahora bien, la perspectiva otra sería una apuesta política desde la cual las personas que están por fuera de cualquier posibilidad de representar insisten, animan y remueven los contenidos que los han silenciado y la desvalorizado. La consideración de una perspectiva otra se puede sostener a partir de lo que propuso el filósofo de la Universidad de Rabat en Marruecos, Khatibi (2001), quien sostiene "este pensamiento-otro", el cual se basa en "una estrategia-otra, la que no avanza nada sin volverse contra sus fundamentos. Una estrategia sin sistema cerrado, sino construcción de un juego del pensar y de lo político que gana terreno silenciosamente sobre sus desfallecimientos y sus sufrimientos. Descolonizarse, ésta es la posibilidad del pensamiento" (p. 71).

En este caso, una perspectiva otra sería aquello que no se asume como total, acabado y totalizador, sino que, por el contrario, siempre se parte de la posibilidad de generar conexiones, relaciones, encuentros y emergencias, que son el resultado de un "juego entre el pensar y lo político”. En este sentido, la perspectiva otra ayuda a la formulación y el diseño de una "estrategia otra”, la cual, en el caso de la educación y la escuela, viene a incidir en la orientación y dinamización de la pedagogización. De ahí que la pedagogización como una perspectiva otra se esfuerza por generar un ambientación de reflexión-acción, llegando a tomar en serio "el potencial no incluido por la modernidad" (Dussel, 2004, p. 201).

El desafío de asumir una perspectiva otra en la escuela nos ayuda a reflexionar y escenificar la pedagogización como una instancia de mutua afectación, llegando a considerar aquellos actores, contenidos, prácticas, discursos, representaciones, contextos y textualidades "no-incluidos", "no-existentes" y "no-valorados" en los espacios hegemónicos de producciónintervención, ante lo cual se procura generar espacios de producción que partan de las realidades, es más la acción-reflexionada que guía la experiencia. Para lograr esto, se requiere que la escuela tome en serio su contexto, las necesidades y, sobre todo, asumir lo dejado por fuera de una demarcación que se corresponde con "el pensamiento abismal moderno". 


\section{METODOLOGÍA}

La investigación se realizó desde una perspectiva crítica y su interés se definió alrededor del reconocimiento de las dinámicas contextuales y el papel que juegan los actores. Lo anterior se pudo afrontar bajo el desarrollo de la "investigación con-sentida” (Villa, 2012), con la cual se insistió en el direccionamiento de la acción, es decir, una estrategia de co-alianza entre los participantes, donde tenemos que este tipo de proceso buscó el "con-sentimiento" que consistió en actuar desde, con, entre y para la puesta en marcha de una dinámica que ayudó a identificar y afrontar los problemas de la colectividad. A partir de esto, se insistió en la necesidad de reaccionar contra los estados de naturalización de las creencias, los estereotipos, los prejuicios, las presuposiciones, los imaginarios y todo tipo de representación que se hacen incuestionables.

También se buscó reaccionar contra las transferencias que hacen los agentes externos, quienes intervienen el lenguaje y llegan a institucionalizar las prácticas de nombrar y actuar, tal como sucede con los funcionarios públicos, los delegados de las ONG, los profesionales de las agencias de cooperación, los militantes de los partidos políticos, los integrantes de los sindicatos y aquellos investigadores que son ajenos a las comunidades. "Las ciencias sociales, de manera muy particular la antropología, cuando se enfrenta a la tarea de analizar un sistema de relaciones interétnicas, cualquiera que éste sea, adopta casi siempre de manera implícita o explícita escalas axiológicas según las cuales se califican y se jerarquizan los sistemas sociales culturalmente diferente que están en relación dentro del sistema interétnico" (Batalla, 1997, p. 203). Como es de esperar las elaboraciones que se desprenden de estas "escalas axiológicas", terminan alojándose en los discursos, llegando a incidir en las relaciones cotidianas de las comunidades.

El principal interés de la investigación a propósito de los saberes de negación y las prácticas de afirmación, fue el de comprender-actuar a partir de las posibilidades que brinda la "complementariedad epistemológica” (Arnal, Rincón \& Latorre,
1992, p. 38). Todo esto, teniendo en cuenta la pedagogizados de estos saberes en la escuela, donde se llega a adoptar una perspectiva otra, donde lo central sea el cambio de mentalidad entre las personas. Para lograr este cometido desde la investigación, se hizo un relacionamiento con el paradigma interpretativo o histórico hermenéutico, que invita a la superación de las "nociones científicas de explicación, predicción y control del paradigma positivista por las nociones de comprensión, significado y acción. La perspectiva interpretativa penetra en el mundo personal de los sujetos (cómo interpretan las situaciones, qué significan para ellos, qué intenciones tienen)" (Arnal, et al. 1992, p. 41). Frente al paradigma sociocrítico, este se empleó por el énfasis que pone en la acción-transformación, donde se puede desnudar las ideologías y los mecanismos que inciden en la construcción del conocimiento y la construcción misma de la realidad de los actores sociales. Los principios del paradigma sociocrítico son:

1. Conocer y comprender la realidad como praxis

2. Unir la teoría y la práctica: conocimiento, acción y valores

3. Orientar el conocimiento a emancipar y liberar al hombre

4. Implicar al docente y los sujetos culturales por medio de la autorreflexión (Arnal, Rincón \& Latorre, 1992, p. 41).

El referente que asumimos de la perspectiva crítica no corresponde con la Escuela de Frackfurt, es más un horizonte crítico que busca abordar el "locus de enunciación" como un aspecto que incide en la construcción epistemológica y metodológica, donde tenemos que la geopolítica del conocimiento traza una demarcación universal que está por encime de cualquier localización específica. "Por supuesto que el "yo" que escribe aquí también debe ser pensado en sí mismo como "enunciado". Todos escribimos y hablamos desde un lugar y un momento determinados, desde una historia y una cultura específicas. Lo que decimos siempre está "en contexto", posicionado” (Hall, 2010, p. 281), de ahí el interés y la importancia por el lugar desde donde se dicen las cosas. Ahora bien, lo crítico se asume en relación con el "programa de investigación 
de Modernidad/colonialidad" (Escobar, 2007, p. 11), sobre todo en lo relacionado con:

1. La Diferencia colonial, es un espacio epistemológico y político de privilegio (Escobar, 2007, p. 23).

2. La Colonialidad del poder, un modelo hegemónico global de poder instaurado [...] el cual viene operando desde hace mucho tiempo (Escobar, 2007, p. 24).

3. La Colonialidad del ser, dimensión otológica [...] apunta hacia el "exceso de ontología" que ocurre cuando seres particulares imponen sobre otros y, más allá de estos, la efectividad potencial o actual de los discursos con los cuales el otro responde a la supresión (Escobar, 2007, p. 25).

4. La Hermenéutica pluritópica, una posibilidad de pensamiento desde diferentes espacios que finalmente rompe con el eurocentrismo como la única perspectiva epistemológica (Escobar, 2007, p. 30).

Las perspectivas o paradigmas que se asumieron en la investigación, incidieron en la construcción epistemológica y metodológica. Sobre todo, permitieron un relacionamiento horizontal con las textualidades que producen los sujetos de la investigación, llegando a incidir no solo en los procedimientos, sino en el pensar y actuar en el proceso. Dada la singularidad de la investigación, se hizo necesario apelar a una tipo de diseño flexible que corresponde a la naturaleza de investigación cualitativa. A este respeto, Mendizábal (2006) sostiene:

Un diseño flexible [...] alude solo a «la estructura subyacente» de los elementos que gobiernan el funcionamiento de un estudio [...]; se refiere a la articulación interactiva y sutil de estos elementos que presagian, en la propuesta escrita, la posibilidad de cambio para captar los aspectos relevantes de la realidad analizada durante el transcurso de la investigación. El concepto de flexibilidad alude a la posibilidad de advertir durante el proceso de investigación situaciones nueva e inesperadas vinculadas con el tema de estudio, que pueden implicar cambios en las preguntas de investigación y los propósitos y a la factibilidad de elaborar conceptualmente los datos en forma original durante el proceso de investigación. Este proceso se desarrolla en forma circular; opuesto, por lo tanto, al derrotero lineal unidireccional (p. 67).

La metodología partió de la identificación de textos que representan las personas en los contextos. Estos son los textos institucionales, académicos, culturales, y la posible relación o choque que se da a partir de la interacción en un espacio común. En este caso tenemos que un primer momento fue el de seleccionar los libros, a partir de los cuales se buscó mirar las representaciones sobre los otros, logrando identificar los estereotipos negativos y demás formas de representar que gana terreno en el decir sobre las personas, los pueblos y comunidades. A su vez, frente a la consideración de los textos culturales, estos se abordaron a partir de una perspectiva semiótica que permitió fijar la atención sobre "lo dado, lo creado y lo adquirido" (Zecchetto, 2002 , p. 23), ver tabla 1.

Frente a la observación de las prácticas en los contextos de las escuelas, se observa que aún tienen peso el desarrollo de contenido mediante la representación de lo folclórico. Esto se entiende como un conjunto de expresiones que fosilizan la cultura, y que corresponden más a la necesidad de generar un texto que se pone en escena a través del interés de existir para otro espectador que satisface su curiosidad frente a los étnicos. Lo dicho se refleja en la siguiente frase que le escuchamos a un profesor que intenta implementar las cátedras de estudios afrocolombianos: "en la institución donde trabajo ya estamos implementando la cátedra de estudios afrocolombianos; hemos empezado por montar un grupo de danza. Al menos ya tenemos qué mostrar cuando la ocasión así lo exija; ya tenemos montadas varías danzas”.

Al momento de indagar sobre la acción situada de los saberes, esto se realizó a partir del ejercicio de "investigación con-sentida", donde se apeló a una horizontalidad de saberes, actores y escenarios. En esta dinámica de acción fue 
importante la co-alianza, es decir, la vinculación motivada y motivadora de las personas que participan para resolver o afrontar una problemática en común. De ahí nacieron los talleres y todas las estrategias de trabajo grupal que permitieron afrontar las narrativas "propias" y "apropiadas". Todo esto se hizo a partir del interés por generar una "política de producción de conocimiento que incida en la política de producción cultural”, llegando a incidir en los "control cultural" (Guillermo Bonfil Batalla, 195); se trata de generar un actuar desde adentro y desde abajo, esto es, una proceso de emergencia a partir de la localización de la experiencia (figura 1).

\section{Tabla 1}

Los textos culturales y sus referentes de producción semiótica

\begin{tabular}{|c|c|c|c|}
\hline \multirow{2}{*}{ Localización } & \multicolumn{3}{|c|}{ Referente manifiesto en las dinámica de producción } \\
\hline & Lo dado & Lo creado & Lo adquirido \\
\hline Endógena & $\begin{array}{l}\text { Lo naturalizado } \\
\text { a partir de la } \\
\text { experiencia de } \\
\text { transmisión } \\
\text { cultural; por } \\
\text { ejemplo, "negro } \\
\text { que no le guste el } \\
\text { ron, es negro que } \\
\text { no tiene sabor" }\end{array}$ & $\begin{array}{l}\text { Emergencia } \\
\text { a partir del } \\
\text { relacionamiento. } \\
\text { "No todos los } \\
\text { bunachis son } \\
\text { iguales, porque no } \\
\text { todos piensan lo } \\
\text { mismo de nosotros } \\
\text { los indígenas" }\end{array}$ & $\begin{array}{l}\text { Interculturalizar } \\
\text { escenarios como la } \\
\text { escuela. "Los niños } \\
\text { afrodescendientes } \\
\text { son niños } \\
\text { inteligentes, iguales } \\
\text { a los demás, solo } \\
\text { que se necesitan ser } \\
\text { reconocidos" }\end{array}$ \\
\hline Exógena & $\begin{array}{l}\text { Lo habituado desde } \\
\text { los estereotipos } \\
\text { y prejuicios, } \\
\text { "los indios son } \\
\text { ladrones" }\end{array}$ & $\begin{array}{l}\text { A partir de la } \\
\text { emergencia el } \\
\text { reconocimiento de } \\
\text { los aportes del otro, } \\
\text { desprejuiciamiento. } \\
\text { "Los negros } \\
\text { son gente de } \\
\text { trabajo, sin ellos } \\
\text { las murallas } \\
\text { de Cartagena, } \\
\text { las ciudades de } \\
\text { como Bogotá, no } \\
\text { tuvieran ciudades } \\
\text { coloniales" }\end{array}$ & $\begin{array}{l}\text { "Las niñas indígenas } \\
\text { llegan a la escuela } \\
\text { con los saberes de } \\
\text { la comunidad, sobre } \\
\text { todo con aquellas } \\
\text { que tienen que ver } \\
\text { con la medicina } \\
\text { tradicional" }\end{array}$ \\
\hline
\end{tabular}

Fuente: elaboración propia

Con respecto al trabajo de la pedagogización, se presentan "obstáculos epistemológicos” que se afirman en los saberes que gozan con cierto prestigio. Aún en las escuelas se sigue reproduciendo una pedagogía institucionalizada que busca la eficacia, la eficiencia a través de los discursos de calidad. Esta "impronta formal" (Baquero \& Villa, 2014, p. 45), se manifiesta en la fe ciega a los manuales, las normas y los criterios disciplinares que generan una modelación del cuerpo y la conciencia. También se pudo ver con la metodología el choque de imaginarios que terminan deshaciendo la posibilidad de existencia del otro y lo otro. Para la visibilización de las fases de 
la investigación se construyó la siguiente tabla, que refleja las cuatro fases del proceso desarrollado (tabla 2).

\section{Figura 1}

Política de producción cultural y política de producción de conocimiento

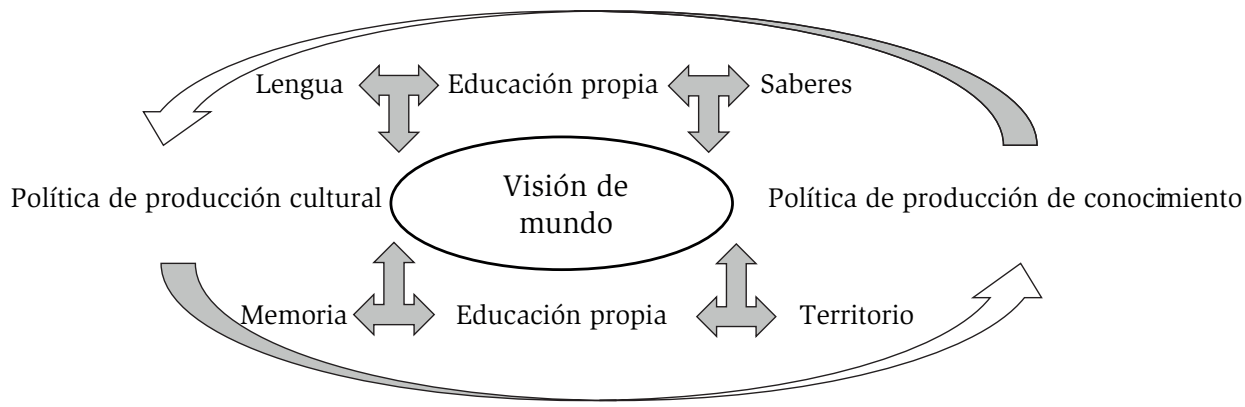

Fuente: elaboración propia

\section{Tabla 2}

Fases de la investigación

\begin{tabular}{|c|c|c|c|}
\hline Fase & Nombre & Actividad & Material analizado \\
\hline \multirow[b]{2}{*}{1.} & \multirow[b]{2}{*}{$\begin{array}{l}\text { Trabajo } \\
\text { interpretativo }\end{array}$} & \multirow{2}{*}{$\begin{array}{l}\text { Análisis de } \\
\text { material que } \\
\text { referencian } \\
\text { el lugar de la } \\
\text { experiencia }\end{array}$} & $\begin{array}{l}\text { Lo dicho: documentos, archivos, } \\
\text { libros y cartillas }\end{array}$ \\
\hline & & & $\begin{array}{l}\text { El decir: textos orales que reflejan } \\
\text { la actualidad de la palabra y su } \\
\text { carácter performativo }\end{array}$ \\
\hline 2. & $\begin{array}{l}\text { Actuando en el } \\
\text { contexto }\end{array}$ & $\begin{array}{l}\text { Talleres de } \\
\text { visibilización }\end{array}$ & $\begin{array}{l}\text { Registros orales } \\
\text { Representación gráfica del } \\
\text { territorio } \\
\text { Exposición de artefactos } \\
\text { culturales que se convierten en } \\
\text { lugares de memoria } \\
\text { Clasificación de saberes } \\
\end{array}$ \\
\hline 3. & $\begin{array}{l}\text { Volviendo sobre } \\
\text { las experiencia }\end{array}$ & $\begin{array}{l}\text { Talleres de } \\
\text { retroalimentación }\end{array}$ & $\begin{array}{l}\text { Presentación de relatorías } \\
\text { Textos audiovisuales } \\
\text { Material fotográfico } \\
\end{array}$ \\
\hline 4. & $\begin{array}{l}\text { Interculturalizar } \\
\text { los encuentros }\end{array}$ & $\begin{array}{l}\text { Trabajo por } \\
\text { grupos focales } \\
\text { desde el } \\
\text { compartir la } \\
\text { experiencia e } \\
\text { interés por los } \\
\text { saberes }\end{array}$ & $\begin{array}{l}\text { Material testimonial a partir de } \\
\text { los mayores, jóvenes, líderes y } \\
\text { lideresas. }\end{array}$ \\
\hline
\end{tabular}

Fuente: elaboración propia 


\section{RESULTADOS}

Este trabajo buscó incidir en los discursos y las prácticas de los y las maestras que abordan su ejercicio desde un "en-lugar”, es decir, una "medida de anclaje donde la conexión con la cotidianidad" es permanente, tal como ha sucedido en algunos contextos con la etnoeducación, que se encuentra reconocida en la Ley 115 o Ley General de Educación, título III del capítulo III que refiere a la "educación para grupos étnicos" (Colombia, Congreso, 8 de febrero de 1994); en el Decreto reglamentario 804 de 1995, que establece el marco general para el desarrollo de la etnoeducación (Colombia, 18 de mayo de 1995). En el caso de la diáspora africana, se cuenta con el Decreto 1122 de 1998 (Ministerio de Educación Nacional, 2001). Este se deriva de la Ley 70 de 1993 o Ley de Comunidades Negras, en especial el capítulo VI, que desarrolló los "Mecanismos para la protección y desarrollo de los derechos y de la identidad cultural" (Colombia, 27 de agosto de 1993).

Frente a la educación de los pueblos indígenas, se parte de un marco común que es regulado por el Estado a través de la etnoeducación. Esto tienen que ver con los referentes citados anteriormente, pero ya en la práctica se vienen desarrollando los procesos de "educación propia", que "se encuentran en sintonía con la resistencia que los pueblos indígenas vienen realizando desde adentro, con el trabajo de los mayores y la comunidad, es totalmente diferente a la etnoeducación que también las prácticas los maestros indígenas en las escuelas”. Esto lo manifestó Walter Izquierdo, un profesor arhuaco de la Sierra Nevada de Santa Marta, en un taller de etnoeducación en la Escuela Normal Superior de Manaure Cesar. Además, esto se relaciona con lo señalado por el profesor Abelardo Ramos Pacho, indígena nasa de Tierradentro: "sentimos que la etnoeducación se quedó a medio camino cuando se burocratizó; por ese motivo sentimos que la educación propia es lo nuestro, es la resistencia por la lengua, la memoria y el territorio".

El problema que se presenta con algunas escuelas del área es que se cree que la etnoeducación es solo traducir los saberes convencionales a la lengua de los otros. Esta es una medida que limita el actuar del maestro, quien cree que solo es hacer equivalencias al sistema de signos establecidos, y esto se hace a partir de simular un ejercicio de reflexión e inclusión de las personas que forman parte de la comunidad, quienes tienen la obligación de acompañar los procesos y también participar de estos. Frente a la posibilidad de la pedagogización de la oralidad en contexto de afirmación cultural, se sigue empleando como un medio para llegar a un fin, y no se ve como un fin que ayuda a desarrollar procesos.

A partir de la pedagogización se pudo hacer una reflexión sobre lo que se encarga de hacer la escuela, así como lo que le corresponde a la comunidad y la familia asumir; con esto se llegó a la conclusión de que no todo se puede curricularizar. De ahí que existan unos ámbitos de la vida comunitaria que no se pueden llevar a la escuela, ya que estos ámbitos de producción son más restringidos al proceso de socialización en la familia y no de la sociabilidad que se genera a partir de los espacios comunitarios.

Durante el proceso de investigación reflexionamos sobre la etnoeducación, su desarrollo y las posibilidades, así como la educación propia. Ante esta situación, preferimos respetar las posturas y decisiones adoptadas por los colectivos de profesoras y profesores. En este caso se consideró que lo importante no es la denominación, sino las apuestas que llevan a la movilización de ciertos saberes y prácticas. Por eso se trató de valorar la disposición y el compromiso de las y los profesores, quienes con su labor y experiencias actúan en la significación y resignificación del papel de la educación, la escuela y la figura del docente como "investigadores de la cultura"; con ello se logra deshacer el lugar de privilegio de la investigación y los investigadores que encarnan el "punto cero", es decir, un lugar seguro para representar una neutralidad afirmada desde la posibilidad de mirar u otear desde un punto superior, el cual "asume producir un conocimiento superior por no estar contaminado por nada terrestre (ni social, espacial, temporal o corporal)" (Grosfoguel, 2013, p. 37). Por el contrario, se trata de generar procesos colaborativos, 
cooperativos y participativos, en los que la emergencia sea el resultado de una co-alianza.

Más allá de la denominación que proviene de la etnoeducación y la educación propia, resaltamos las experiencias, sobre todo que independientemente la denominación, lo que importa es la posibilidad de producir conocimiento desde adentro y desde abajo. Ante esto manifestamos que las dos se convierten en una oportunidad para generar conocimiento a partir de lo intracultural. Por este motivo, se hace necesario insistir en la "la pedagogización de la escucha a través de la conversación intergeneracional” (Villa \& Villa, 2013). Desde este referente se pueden considerar los siguientes resultados:

- Los participantes del proceso adquirieron herramientas metodológicas para analizar los saberes de negación, todo esto a partir de los contenidos textuales que involucran lo dicho y el decir.

- Se afrontaron los imaginarios, y con ello se llegó a valorar la reproducción y la producción de estereotipos negativos, así como positivos.

- A partir de las representaciones se analizó el problema de la folclorización, así como la espectacularización de las producciones simbólicas y materiales de los pueblos, las comunidades y las colectividades.

- Teniendo en cuenta los procesos de pedagogización en la escuela y la comunidad, se entró a valorar la educación con pertinencia cultural, sobre todo el papel que desempeñan las y los profesores en la revitalización de saberes y prácticas locales.

- Desde el hecho de afrontar los problemas que inciden en los procesos de autoafirmación, se abordó el papel que cumple la autoimagen.

- Se identificó el papel que tiene el marco regulativo del Estado, sobre todo en lo relacionado con el derecho étnico de los pueblos.

- Las y los profesores identifican como necesaria una política de producción de conocimiento que actúe en la afirmación cultura desde la educación y la escuela.

Finalmente, creemos necesario resaltar el papel del lenguaje en todo este proceso. A partir de este se pudo generar un interés por la forma como las personas articulan la experiencia, con lo cual se llegó a afrontar las dinámicas de negación en medio de las prácticas de afirmación cultural, en la que saberes y prácticas dan sentido a la experiencia comunitaria. También se actuó desde el lenguaje especialmente en lo que tiene que ver con las narrativas de invención, las cueles aluden a las vivencias intra- e interculturales.

\section{CONCLUSIÓN Y DISCUSIÓN}

A partir de la relación entre la teoría y la práctica se pudieron abordar los procesos de negación y la constitución de imaginarios que inciden en la representación de las personas, tanto en la educación, la escuela y la comunidad. También se abordaron las expresiones que actúan en la organización significativa de la realidad, y con esto se establecieron unos referentes críticos que acompañen la reflexión de los profesores y los demás miembros de la comunidad; esto con el fin de asumir de forma creativa con que se vienen desarrollando las apuestas por la afirmación cultural.

El asumir la escuela desde una perspectiva otra, nos lleva a valorar los procesos de "en-lugar", donde se asume la pedagogización de la tradición oral, la reconstrucción del territorio, los saberes y las prácticas de sujeción a la memoria, las formas solidarias de producción e intercambio de alimentos, las fechas y celebraciones que exaltan la experiencia colectiva. De ahí que se vea la escuela como una institución que actúa de puertas abiertas a la comunidad. En este sentido, la escuela se toma como un proyecto que está en permanente construcción, el cual debe de responder a los procesos intra- e interculturales. En cuanto a la producción de conocimiento, surge la necesidad de insistir en la acción situada, esto es, una preocupación por las formas de dinámicas de producción y distribución del conocimiento, donde "el con-sentimiento", es decir, un actuar desde, con, entre y para sea lo central. En este caso, se requiere del reconocimiento de la textualidad cultural que escenifican los sabedores y las sabedoras a partir de las narrativas locales. Por todo lo expuesto en los párrafos anteriores, queremos presentar a continuación las siguientes conclusiones: 
- Se resalta el papel que desempeñan las narrativas en la elaboración, la circulación y la distribución de los saberes de negación, así como las prácticas de afirmación.

- A partir de la etnoeducación y la educación propia se llegó valorar las dos como una instancia generadora de una política de producción de conocimiento al servicio de la comunidad, más allá de las diferencias en la definición o forma de asumir cada una.

- Considerar el diálogo intergeneracional como una posibilidad de pedagogizar la voz interior de las comunidades, todo esto a partir de los procesos intraculturales e interculturales.

- Se valoró la frontera como un espacio vital que permite los encuentros y por ello la emergencia de saberes que son negociados, problematizados, rechazados y en algunos casos aceptados como apropiados.

- Ver los saberes de negación como un tipo de producción que le disputa el espacio de articulación y generación de sentido a las prácticas de afirmación.

Finalmente, el esfuerzo por generar una política de producción de conocimiento debe propender a una "política de la voz" (Giroux, 2003), es decir, una política de afirmación de las personas que son silenciadas, negadas, vituperadas y desterradas de cualquier posibilidad de construir su propia imagen. La "política de la voz" se puede abordar desde las narrativas culturales, que al ser pedagogizadas se puede incidir en la autoproyección de una colectividad que se represente como portadora de valores.

\section{REFERENCIAS BIBLIOGRÁFICAS}

Alarcón, L., A, Calderón, J., \& Santos, A. (2002). Educación y cultura en el Estado Soberano del Magdalena. Barranquilla: Fondo de Publicaciones Universidad del Atlántico.

Anderson, B. (1997). Comunidad imaginada. México: Fondo de la Cultura Económica.

Arnal, J, Rincón, D., \& Latorre, A (1992). Investigación educativa. Barcelona: Labor.

Batalla, B. G. (1997). "Implicaciones éticas del sistema de control cultural”. Ética y diversidad cultural. México: Fondo de la Cultura Económica.
Baquero, P., \& Villa, W. (2014). Un visitante en el aula. Bogotá: Universidad Distrital Francisco José de Caldas.

Berger, P., \& Luckmann, T. (1995). Modernidad, pluralismo y crisis de sentido. Barcelona: Paidós.

Boia, L. (1997). Entre el ángel y la bestia. Santiago de Chile: Andrés Bello.

Castro, G. S. (2010). La Hybris del punto cero. Bogotá: Pontificia Universidad Javeriana.

Colombia (27 de agosto de 1993). Ley 70 de 1993, por la cual se desarrolla el artículo transitorio 55 de la Constitución Política. Diario Oficial 41.013. Bogotá.

Colombia (18 de mayo de 1995). Decreto 804 de 1995, por medio del cual se reglamenta la atención educativa para grupos étnicos. Diario Oficial 41.853. Bogotá.

Colombia, Congreso (8 de febrero de 1994). Ley 115 de 1994, por la cual se expide la Ley General de Educación. Diario Oficial 41.214. Bogotá.

Dussel, E. (2004). "Sistema-mundo y transmodernidad”. Modernidades coloniales. México: El Colegio de México.

Eisner, E. W. (1995). Educar la visión artística. Barcelona: Paidós Educador.

Escobar, A. (2007). "Mundos y conocimiento de otro modo" El programa de investigación de modernidad/colonialidad latinoamericano. Educación superior, interculturalidad y descolonización. La Paz: Programa de Investigación Estratégica en Bolivia.

Giroux, E. (2003). Pedagogía y política de la esperan$z a$. Madrid: Amorrortu.

Grosfoguel, R. (2006). "La actualidad del pensamiento de Césaire: redefinición del sistema-mundo y producción de utopía desde la diferencia colonial”. Discurso sobre el colonialismo. Madrid: Akal.

Grosfoguel, R. (2013). Racismo/sexismo epistémico, universidades occidentalizadas y los cuatros genocidios/epistemicidios del largo siglo XVI. Tabula Rasa, (27). Bogotá: Universidad Colegio Mayor de Cundinamarca.

Herbart, J. F. (1935). Pedagogía general derivada del fin de la educación. Madrid: La Lectura.

Hall, S. (2010). Sin Garantías: trayectorias en estudios culturales. Bogotá: Instituto Pensar-Pontificia Universidad Javeriana.

Khatibi, A. (2001). "Maghreb plural”. Colonialidad del poder. Cultura y conocimiento en América Latina. Capitalismo y geopolítica del conocimiento. Buenos Aires: Signo.

McGraw, J. (2007). Purificar la nación: eugenesia, higiene y renovación moral-racial de la periferia del Caribe colombiano, 1900-1930. Revista de Estudios Sociales, (27), 67-75. 
Mendizábal, N. (2006). Los componentes del diseño flexible en la investigación cualitativa. Estrategias de investigación cualitativa. Barcelona: Gedisa.

Merani, A. (1985). Diccionario de pedagogía. México: Grijalbo.

Ministerio de Educación Nacional (2001). Lineamientos curriculares. Cátedra Estudios Afrocolombianos. Bogotá.

Quijano, A. (2001). Colonialidad del poder. Cultura y conocimiento en América Latina. Capitalismo y geopolítica del conocimiento. Buenos Aires: Signo.

Sáenz, J., Saldarriaga, O., \& Ospina, A. (1997). Mirar la infancia: pedagogía, moral y modernidad en Colombia, 1903 a 1946. Bogotá: Foro Nacional por Colombia.

Santos, B. S. (2009). Una epistemología del Sur. México: Siglo XXI.

Villa, W. (2012). Los silencios que se prolongan. La representación de los otros en la investigación. Universidad, epistemología emergente y metodología en otredad. Bogotá: Universidad Libre.

Villa, W. (2013). Memoria y pedagogización del mal-decir: una aproximación a los recorridos que inventan mundos. Cuadernos de Filosofía de Latinoamérica, 34 (108). Bogotá: Universidad Santo Tomas.
Villa, W. (2014a), Lenguajes del silencio. Ausenciapresencia de los afros en los Andes. Johannesburgo: Instituto CASAS y Universidad de Witwatersrand.

Villa, W. (2014b) La emergencia de lo Caribe y el silenciamiento de las comunidades negras. Bogotá: Organización de Raizal de Residentes Fuera del Archipiélago de San Andrés, Providencia y Santa Catalina ORFA.

Villa, W \& Villa, E (2010). La pedagogización de la oralidad en contexto de afirmación cultural de las comunidades negras del Caribe seco colombiano. Cuadernos de Literatura del Caribe e Hispanoamérica, (12), 69-89.

Villa, W \& Villa, E (2013). Donde llega uno, llegan dos, llegan tres y llegan todos. El sentido de la pedagogización de la escucha en las comunidades negras del Caribe Seco Colombiano. Pedagogías decoloniales. Quito: Abya Yala.

Walsh, C. (2007). "(De)colonialidad e interculturalidad epistémica: política, ciencia y sociedad de otro modo". Educación superior; interculturalidad y descolonización. La Paz: Programa de Investigación Estratégica en Bolivia.

Zecchetto, V. (2002). La danza de los signos. Quito: Abya Yala.

Zuluaga, O. (1987). Pedagogía e historia. Bogotá: Foro Nacional por Colombia. 